\title{
Scaling in activated escape of underdamped systems
}

\author{
M.I. Dykman \\ Department of Physics and Astronomy, Michigan State University, East Lansing, MI 48824, USA \\ I.B. Schwartz \\ Plasma Physics Division, Code 6792, Naval Research Laboratory, Washington DC 20375 \\ M. Shapiro \\ Department of Mathematics, Michigan State University, East Lansing, MI 48824, USA
}

\begin{abstract}
Noise-induced escape from a metastable state of a dynamical system is studied close to a saddlenode bifurcation point, but in the region where the system remains underdamped. The activation energy of escape scales as a power of the distance to the bifurcation point. We find two types of scaling and the corresponding critical exponents.
\end{abstract}

PACS numbers: 05.40.-a, 05.70.Ln, 85.25.Cp, 77.65.Fs

\section{INTRODUCTION}

Noise-induced escape from a metastable state underlies many important phenomena in nature and technology. It is often investigated experimentally for systems close to a bifurcation point where a metastable state disappears. Examples include Josephson junctions [1, 2, 3], nanomagnets [4, 5, 6], and mechanical nano- and microresonators [7, 8, 9]. Studying escape in this regime is advantageous in two respects. First, near a bifurcation point the activation barrier is small, so that the escape rate can be measured in a conceivable time even for low temperature or small noise intensity. Second, the dynamics near a bifurcation point displays system-independent features, including the onset of one or several soft modes. In turn, this leads to scaling of the escape rate with the control parameter of the system.

We will discuss escape for system near a generic saddlenode bifurcation, which is one of the most common types of bifurcations. Here, for very small values of the control parameter $\eta$ counted off from the bifurcation value $\eta_{B}=0$, the metastable and unstable states of the system are close to each other in phase space. The motion along the axis that connects them is slow. This is easy to understand when metastable and unstable states are a minimum and a saddle point of the potential energy of a particle. The curvature of the potential has opposite signs at these points. Therefore the potential locally flattens out as $\eta$ approaches zero. In agreement with this picture, for very small $\eta$ the system dynamics is controlled by a single soft mode $q$, which is overdamped.

In this paper we consider systems with very small damping. Their motion may become underdamped even where the parameter $\eta$ is still close, although not too close to the bifurcation value. The dynamics of the soft mode is characterized by the vibrational frequency at the metastable state $\omega_{a}$. It is much less than other frequencies, and $\omega_{a} \rightarrow 0$ for $\eta \rightarrow 0$. Yet $\omega_{a}$ may exceed the relaxation rate, which is characterized by an independent small parameter $\varepsilon$. Such a situation often occurs in underdamped Josephson junctions, for example.

Noise-induced escape near a bifurcation point is well understood in the limit $\eta \rightarrow 0$, where the motion is overdamped 10, 11, 12, 13]. Here, under fairly general assumptions the activation energy of escape scales as $\eta^{3 / 2}$. This agrees with the scaling of the mean-field free-energy barrier near a termination point of the metastable state 14]. As we show, such scaling holds also for underdamped systems further away from $\eta=0$ provided the metastable and saddle states are still close to each other. This can be understood for a Brownian particle in a potential well. Here, the activation energy of escape is given by the height of the potential barrier, independent of friction; friction only affects the prefactor in the escape rate [15]. Therefore the scaling is the same in the under- and overdamped regimes. The scaling $\eta^{3 / 2}$ was discussed already in the early work on Josephson junctions 16 and later for underdamped driven oscillators [17] and magnets 18 .

As we show, besides the $\eta^{3 / 2}$ scaling, underdamped systems may display a different scaling near, but not too close to the bifurcation point. This is because the motion slowing down is not necessarily related to the stable and unstable state approaching each other. The slowing down may have a different, nonlocal origin. It can be thought of as resulting from flattening, or an overall decrease in magnitude of the effective potential of the system in a broad range of the generalized coordinate. We provide a theory of escape for this nonlocal case and find the corresponding critical exponent. The results are applied to a specific system, which provides an example of this scaling behavior and has attracted much attention recently [3, 7, 8, 9].

In Sec. II we discuss the model of a fluctuating underdamped system. In Sec. III we describe two scenarios that lead to the motion slowing down and ultimately to 
the saddle-node bifurcation, in the presence of friction, as the control parameter $\eta \rightarrow 0$. The effect of friction is described in Sec. IV. Energy diffusion is considered in Sec. V. In Sec. VI we obtain the critical exponents for the activation energy of escape, which is the central result of the paper. In Sec. VII the theory is applied to a nonlinear oscillator driven by a strong resonant field and compared with the previous results for this model [17, 19]. Sec. VIII contains concluding remarks.

\section{THE MODEL}

Close to a saddle-node bifurcation point one of the motions in the system becomes slow compared to other motions. Quite generally it is described by a Langevin equation

$$
\begin{aligned}
& \dot{q}=\partial_{p} H(p, q)-\varepsilon v^{(q)}(p, q)+f^{(q)}(t), \\
& \dot{p}=-\partial_{q} H(p, q)-\varepsilon v^{(p)}(p, q)+f^{(p)}(t) .
\end{aligned}
$$

Here, $q$ and $p$ are the coordinate and momentum of the slow motion, and $H$ is its effective Hamiltonian. The terms $\left.\varepsilon v^{(q, p}\right)$ and $f^{(p, q)}$ describe friction and noise, respectively. We have assumed that the motion is slow on the scale of the correlation time of the reservoir coupling to which leads to friction and noise. Therefore we disregard retardation in the friction force. In the same approximation we disregard the noise correlation time and set the noise to be $\delta$-correlated in time,

$$
\left\langle f^{(i)}(t) f^{(j)}\left(t^{\prime}\right)\right\rangle=2 D_{i j}(p, q) \delta\left(t-t^{\prime}\right),
$$

where $i, j$ enumerate the $q$ and $p$ components of noise. The diffusion matrix is symmetric, $D_{q p}=D_{p q}$, and generally $D_{i j}$ depends on the dynamical variables $q, p$. The noise intensity $\propto \max D_{i j}$ will be the smallest parameter of the theory.

We assume that the friction coefficient $\varepsilon$ is small and that the friction force is non-Hamiltonian. The latter requires that

$$
\nabla \mathbf{v} \equiv \partial_{q} v^{(q)}+\partial_{p} v^{(p)}
$$

is not equal to zero. The part of the force $\left(v^{(q)}, v^{(p)}\right)$ for which $\boldsymbol{\nabla} \mathbf{v}=0$ can be incorporated into the Hamiltonian, leading to its small renormalization $\propto \varepsilon$. This concerns, in particular, $q$-independent terms in $v^{(q)}$ and $p$-independent terms in $v^{(p)}$.

The Hamiltonian $H(p, q) \equiv H(p, q ; \eta)$ depends on the control parameter $\eta$ which characterizes the distance to the bifurcation point. This point is located at $\eta=0$, for $\varepsilon=0$. We require that (i) $H(p, q ; \eta)$ be analytic in $\eta$ in a neighborhood of $\eta=0$, and (ii) $\partial_{\eta} H \not \equiv 0$ for $\eta=0$. These two conditions determine the order of the Hamiltonian $H$ with respect to $\eta$ uniquely for the different functional forms of $H$ used below, i.e., if both $\eta_{1}$ and $\eta_{2}$ meet them, then $\eta_{2}=O\left(\eta_{1}\right)$.
The physical picture of motion leading to escape is simple. The system is initially prepared at some point $(q, p)$ in the basin of attraction to the metastable attractor (focus) $\left(q_{a}, p_{a}\right)$. Over the relaxation time $t_{\mathrm{r}} \propto \varepsilon^{-1}$, the system approaches the attractor. Noise leads to small fluctuations about the attractor, but for small noise intensity the system stays close to it for a time that largely exceeds $t_{\mathrm{r}}$. Ultimately there happens a large fluctuation that carries the system over the basin boundary leading to escape from the metastable state.

\section{A. Brownian particle near a saddle-node bifurcation point: standard analysis}

The probability $W$ of noise-induced escape from a metastable state is a complicated function of the dynamical parameters. However, it displays a universal behavior in the vicinity of a bifurcation point. This behavior has been well understood in two limiting cases. The best known case is a Brownian particle in a potential well, which escapes from the well due to thermal fluctuations. The motion of a Brownian particle of mass $m=1$ in a potential $U(q)$ is described by the equation (10) with

$$
\begin{aligned}
& H(p, q)=\frac{1}{2} p^{2}+U(q), \quad f^{(q)}=0, \\
& v^{(q)}=0, \quad v^{(p)}=p,
\end{aligned}
$$

and with $f^{(p)}(t)$ being white noise of intensity $D=2 \varepsilon k T$.

The dynamics of a Brownian particle is special in several respects. First, the value of the momentum in a stationary state is independent of the parameters and is equal to zero. Second, independent of the friction coefficient, the stable and unstable states of the system $q_{a}$ and $q_{s}$ are the local minimum and maximum of $U(q)$. Third, as a consequence of the form of the noise, the escape rate has a simple form

$$
W=\text { const } \times e^{-\Delta U / k T}, \quad \Delta U=U\left(q_{s}\right)-U\left(q_{a}\right) .
$$

The exponential factor here is just the ratio of the Boltzmann factors in the populations of states with energies of the saddle point and the attractor. It is independent of the friction coefficient. In contrast, the prefactor in $W$ depends on $\varepsilon$ [15]; however, in this work we will be interested only in the exponent in $W$.

The potential $U(q)$ depends on the control parameter $\eta$. The standard analysis of this dependence goes as follows. When $\eta$ is close to the critical value $\eta=0$, the stationary states are close to each other and ultimately merge for $\eta=0$; we set $q_{a}=q_{s}=0$ for $\eta=0$. For small $\eta$ the potential is a cubic parabola, $U(q) \approx U_{\text {cub }}(q)$,

$$
U_{\text {cub }}(q)=-q^{3} / 3+\eta q
$$

locally in $q$ (which has been appropriately scaled). The stable and unstable states are $q_{a}=-\eta^{1 / 2}, q_{s}=\eta^{1 / 2}$. Both the distance $q_{s}-q_{a}$ and the frequency of vibrations 
about the potential minimum in the absence of friction $\omega_{a}=(4 \eta)^{1 / 4}$ decrease with decreasing $\eta$, as expected.

Eq. (6) leads to the scaling of the activation barrier with $\eta$ of the form $\Delta U=(4 / 3) \eta^{3 / 2}$. We emphasize that this scaling holds independent of friction, for a Brownian particle. Because the friction coefficient $\varepsilon$ is small, the approximation (6) applies both where the system is underdamped, with $\omega_{a} \gg \varepsilon$, or underdamped, with $\omega_{a} \ll \varepsilon$. The critical value of the bifurcation parameter $\eta$ is also independent of $\varepsilon$.

\section{B. Close vicinity of the bifurcation point}

Irrespective of the friction coefficient, very close to the bifurcation point the dynamics of the system is overdamped 20]. It is described by a soft mode $Q$, which is the distance between the saddle and the attractor. The Langevin equation for $Q$ after proper scaling and changing to a slow time $\tau$ can be written as

$$
\frac{d Q}{d \tau}=Q^{2}-\eta+f^{(Q)}(\tau)
$$

For $\eta>0$ the system has a stable and unstable states $Q=\mp \eta^{1 / 2}$, which merge together for $\eta=0$.

Because the motion near the bifurcation point is slow, with characteristic time $\eta^{-1 / 2}$, and occurs in a narrow range $|Q| \lesssim \eta^{1 / 2}$, the noise $f^{(Q)}(\tau)$ can be considered white, with a $Q$-independent intensity $D$. Then the escape rate is 10]

$$
W=\frac{\eta^{1 / 2}}{\pi} e^{-R / D}, \quad R=\frac{4}{3} \eta^{3 / 2} .
$$

Eqs. (7), (8) can be understood as the limit of Brownian motion for $\varepsilon \gg \eta^{1 / 4}$, where the Brownian motion becomes overdamped. Eq. (7) follows from Eqs. (11), (4) in this limit, with $Q=q$ and $\tau=\varepsilon t$. The activation energy of escape $R$ (8) scales with $\eta$ in the same way as the barrier height $\Delta U$ for the corresponding Brownian particle.

We emphasize that the applicability of Eq. (7) is not at all limited to systems close to thermal equilibrium. It applies essentially to any weakly fluctuating dynamical system close to a saddle-node bifurcation point, see 13] and references therein. The scaling $R \propto \eta^{3 / 2}$ has been considered a benchmark of activated escape near a saddle-node bifurcation point. It has been used very broadly, in particular as a tool for measuring critical current of Josephson junctions [1, 2].

\section{SADDLE-NODE BIFURCATION IN UNDERDAMPED NONEQUILIBRIUM SYSTEMS}

We will consider the general case of a system away from thermal equilibrium, which is described by Eq. (11) with small friction coefficient $\varepsilon$. Our major assumption is that, as we decrease the control parameter of the Hamiltonian $\eta$ from a positive value $\sim O(1)$ while keeping $\varepsilon=$ const, the system will experience a saddle-node bifurcation. Because the system is nonequilibrium, the bifurcation value $\eta_{B}$ may depend on the friction parameter $\varepsilon$. For convenience, in what follows we do not set $\eta_{B}=0$, but we assume

$$
\left|\eta_{B}\right| \ll 1, \quad \lim _{\varepsilon \rightarrow 0} \eta_{B}=0 .
$$

For small $\varepsilon$ the system is underdamped for $\eta_{B} \ll$ $\eta \ll 1$. Still very close to $\eta_{B}$ it invariably becomes overdamped and is described by Eq. (77). We want to find the forms of the Hamiltonian and the corresponding phase portraits of the Hamiltonian trajectories that will be compatible with this scenario of the saddle-node bifurcation.

By assumption, the bifurcation results from the change of the Hamiltonian dynamics, not from the special structure of the friction force. Then a necessary condition is that the Hamiltonian system has a center $\left(q_{c}, p_{c}\right)$ and a saddle point $\left(q_{s}, p_{s}\right)$ for $\eta>0$. With weak friction, the center becomes a focus. When $\eta$ becomes very close to $\eta_{B}$ the focus becomes a node and ultimately merges with the saddle point.

Central to the occurrence of a bifurcation is the slowing down of the system with decreasing $\eta$. We note that this slowing down should occur not only near the center, but in the whole range of energies between the energies of the center $E_{c}=H\left(q_{c}, p_{c} ; \eta\right)$ and the saddle point $E_{s}=$ $H\left(q_{s}, p_{s} ; \eta\right)$. We will discuss two possible phase portraits that meet this condition. They are shown in Fig. 1]

\section{A. Local Hamiltonian bifurcation}

The simplest evolution of the phase portrait of Hamiltonian dynamics corresponds to the center and the saddle point approaching each other in phase space with decreasing $\eta$ and ultimately merging for $\eta=0$, see upper panel in Fig. 1 We call this "local Hamiltonian bifurcation". The frequencies of the Hamiltonian trajectories surrounding the center $\omega(E)$ decrease with $\eta \rightarrow 0$, because these trajectories are pressed against the homoclinic trajectory which has zero frequency.

This case is easy to analyze. We expand the Hamiltonian $H$ in $q, p$ counted off from their value at the degenerate point into which the center and the saddle point merge for $\eta=0$. Because of the degeneracy, the determinant $\partial_{q}^{2} H \partial_{p}^{2} H-\left(\partial_{q} \partial_{p} H\right)^{2}=0$ at this point. We choose $q$ along the zero-eigenvalue eigenvector of the matrix of second derivatives of $H$ for $\eta=0$. Then to leading order in $q, p, \eta$ the expansion of $H$ takes the form

$$
H(p, q ; \eta) \approx \frac{1}{2} p^{2}+U_{\mathrm{cub}}(q)
$$

with $U_{\text {cub }}(q) \equiv U_{\text {cub }}(q ; \eta)$ given by Eq. (6). 

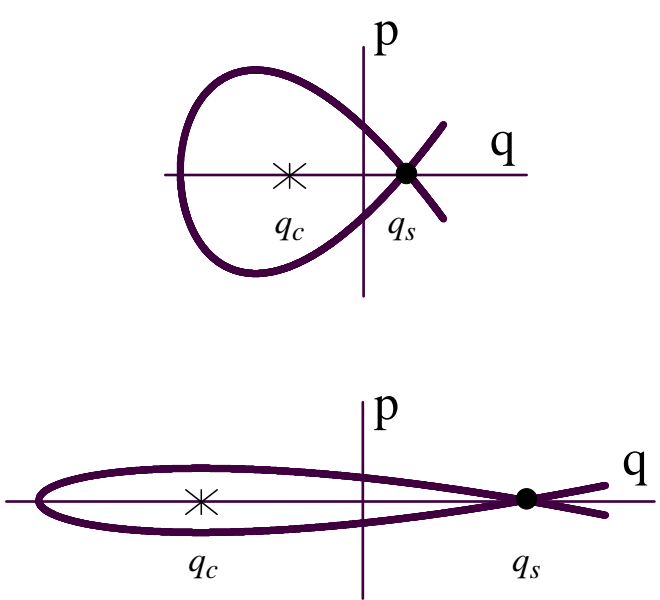

FIG. 1: Two types of phase portraits leading to a saddle-node bifurcation in the presence of dissipation. The homoclinic trajectories go through the saddle point $\left(q_{s}, p_{s}=0\right)$. The center $\left(q_{c}, p_{s}=0\right)$ [attractor, in the presence of dissipation] is shown by the cross. As the control parameter of the Hamiltonian $\eta$ approaches the critical value, the loop in the left panel shrinks in all directions. The center and the saddle approach each other in phase space and ultimately merge at the bifurcation point. The frequency of vibrations about the center $\omega_{a}$ decreases, because the center becomes closer to the homoclinic trajectory, which has an infinite period. In the right panel, the homoclinic orbit also shrinks as $\eta \rightarrow 0$, but the stationary states remain separated. Still the frequency $\omega_{a}$ decreases with decreasing $\eta$, because the trajectories are pressed against the homoclinic orbit.

In Eq. (9) we appropriately scaled $q, p$ and disregarded all cubic terms except for the terms $\propto q^{3}$ and all terms $\propto \eta$ except for $\eta q$. An estimate $|q| \sim \eta^{1 / 2},|p| \sim$ $\eta^{3 / 4}, \omega(E) \propto \eta^{1 / 4}$ shows that the higher-order terms in $q, p, \eta$ lead to small corrections for $\eta \ll 1$. In order to make the results more intuitive, we chose the coefficient at $p^{2}$ to be positive. In this case the center $q_{c}=-\eta^{1 / 2}, p_{c}=0$ has lower energy than the saddle point $q_{s}=\eta^{1 / 2}, p_{s}=0$. If we chose $H=-p^{2} / 2+U_{\text {cub }}(q)$ it would be the other way around, as we illustrate on the example below, but this does not change the following arguments.

In the approximation (9) the homoclinic loop that surrounds the center has a simple form

$$
p= \pm\left(\frac{2}{3} \eta^{3 / 2}-\eta q+\frac{1}{3} q^{3}\right)^{1 / 2} .
$$

The vibration frequency about the center is $\omega_{a}=(4 \eta)^{1 / 4}$. As expected, $\omega_{a} \rightarrow 0$ for $\eta \rightarrow 0$. The difference of the energy values in the extreme points

$$
\Delta E \equiv H\left(q_{s}, p_{s} ; \eta\right)-H\left(q_{c}, p_{c} ; \eta\right)=\frac{4}{3} \eta^{3 / 2} .
$$

\section{B. Nonlocal Hamiltonian bifurcation}

Slowing down in the Hamiltonian system occurs also if the homoclinic trajectory shrinks down with $\eta \rightarrow 0$ while the center $\left(q_{c}, p_{c}\right)$ and the saddle point $\left(q_{s}, p_{s}\right)$ do not move or move only slightly, see lower panel in Fig. 1 As in the local case, the frequencies of all trajectories inside the homoclinic loop go to zero for $\eta \rightarrow 0$. We call it a nonlocal Hamiltonian bifurcation. It corresponds to the homoclinic orbit becoming degenerate, with no motion along it at all, at any point. The values of the Hamiltonian at the center and the saddle point coincide, $H\left(q_{c}, p_{c} ; \eta=0\right)=H\left(q_{s}, p_{s} ; \eta=0\right)$.

We choose the momentum $p$ as the variable along which the trajectories are shrinking for $\eta \rightarrow 0$. This variable is limited by the "height" of the homoclinic loop in Fig. [1 and therefore it is small for small $|\eta|$. Since on the degenerate homoclinic trajectory $\dot{q}=\partial_{p} H=0$ for $\eta=0$, after a proper rescaling of variables the Hamiltonian for small $\eta, p$ takes the form

$$
H(p, q ; \eta)=\frac{1}{2} p^{2}+\eta U(q) .
$$

A more general form

$$
H=\frac{a_{1}^{2}(q)}{2}\left[p-\eta^{1 / 2} a_{2}(q)\right]^{2}+\eta U(q)
$$

is reduced to Eq. (12) by a transition to canonical variables $p^{\prime}=a_{1}(q)\left[p-\eta^{1 / 2} a_{2}(q)\right], q^{\prime}=\int d q / a_{1}(q)$ (we assume that $a_{1}$ does not have zeros in the range of interest).

The specific form of the potential $U(q)$ is not important, except that $U(q)$ must have a local minimum and maximum. For $\partial_{p}^{2} H>0$ [as chosen in Eq. (12)], the minimum of $U(q)$ corresponds to the center $q_{c}$, and the maximum corresponds to the saddle point $q_{s}$. The homoclinic trajectory goes around $q_{c}$, and therefore the turning point of this trajectory $q_{t}$ (where $p=0$ ) lies on the opposite side from $q_{c}$ with respect to $q_{s}$, as in Fig. [1] We assume, in agreement with the qualitative picture of approaching the bifurcation that, in the interval $\left[q_{t}, q_{s}\right]$, the potential has no other extrema besides $q_{c}$ and has no singularities for real $q$.

A simple form of $U(q)$ consistent with the above conditions is a cubic parabola, $U(q)=-q^{3} / 3+q$. In this case $q_{c}=-1, q_{s}=1$, and the turning point $q_{t}=-2$ independent of $\eta$. However, since the distance between $q_{c}$ and $q_{s}$ does not decrease with decreasing $\eta$, there are no reasons to believe that the potential is generically a cubic parabola. We will give an example of a different potential below.

For the Hamiltonian (12), both the momentum and frequency scale with $\eta$ as $|p| \propto \omega(E) \propto \eta^{1 / 2}$. As expected, $\omega_{a}=\eta^{1 / 2} U^{\prime \prime}\left(q_{c}\right) \rightarrow 0$ for $\eta \rightarrow 0$. To leading order in $\eta$ the energy difference at the saddle point and the center is

$$
\begin{aligned}
\Delta E \equiv & H\left(q_{s}, p_{s} ; \eta\right)-H\left(q_{c}, p_{c} ; \eta\right) \\
& =\eta\left[U\left(q_{s}\right)-U\left(q_{c}\right)\right] .
\end{aligned}
$$


It follows from Eqs. (11), (13), that for the local and nonlocal Hamiltonian bifurcations $\Delta E$ displays scaling behavior with the control parameter, $\Delta E \propto \eta^{\xi}$. However, the scaling exponents $\xi$ are different, $\xi=3 / 2$ and $\xi=1$ in the local and nonlocal cases, respectively.

\section{EFFECT OF FRICTION}

The analysis of friction is particularly simple in the case of a local Hamiltonian bifurcation (91). Generally, one would expect the friction force $v^{(p, q)}$ to be smooth near the point $q=p=0$. For small $|q|,|p|$ it can be expanded to linear terms in $q, p$. Keeping only the terms that are not reduced to renormalization of the parameters of the Hamiltonian, we have

$$
v^{(q)}=a_{q} q, \quad v^{(p)}=a_{p} p .
$$

The center $\left(q_{c}=-\eta^{1 / 2}, p_{c}=0\right)$ becomes a stable state provided $\varepsilon\left(a_{q}+a_{p}\right)>0$. It shifts because of the friction to $\left(q_{a}=-\left(\eta+\varepsilon^{2} a_{p} a_{q} / 4\right)^{1 / 2}, p_{a}=\varepsilon a_{q} q_{a}\right)$. The bifurcation value of the control parameter in the local case is

$$
\eta_{B}^{\mathrm{loc}}=-\varepsilon^{2} a_{p} a_{q} / 4
$$

The analysis of the nonlocal case is somewhat more involved. In this case friction changes the behavior of the system very significantly: while the center and the saddle point remain separated for $\eta \rightarrow 0$ in the Hamiltonian case, because of the friction the corresponding attractor and the saddle point are approaching each other as $\eta$ goes to the bifurcation value $\eta_{B}^{\mathrm{nl}}$.

To understand the constraints on the friction force we note that far from the bifurcation point, $\eta \gg \eta_{B}^{\mathrm{nl}}$, the motion of the system is slowly decaying oscillations. It is characterized by the period-average value $V_{E}$ of the rate of energy change

$$
\dot{E}=-\varepsilon\left(v^{(p)} \partial_{p} H+v^{(q)} \partial_{q} H\right) .
$$

It is convenient to do the averaging by writing the integral over time as an integral over the trajectory $H(p, q ; \eta)=$ $E$, which gives

$$
\begin{array}{r}
V_{E}=-\varepsilon \frac{\omega(E)}{2 \pi} \oint\left(d q v^{(p)}-d p v^{(q)}\right) \\
=-\varepsilon \frac{\omega(E)}{2 \pi} \iint_{A(E)} d q d p \nabla \mathbf{v}
\end{array}
$$

where the divergence of $\mathbf{v}$ is defined by Eq. (3). The circulation is taken along the trajectory $H(p, q ; \eta)=E$, and $A(E)$ is the enclosed region.

We assume that the surface integral in Eq. (16) does not change sign for all energies between the center and the saddle point, $E_{c}$ and $E_{s}$. If the integral is positive (we assume $\varepsilon>0$ ) then the period-average energy monotonously drifts down to its value $E_{c}$ if it was initially between $E_{c}$ and $E_{s}$. This is consistent with the assumption that friction does not lead to extra stationary states. A sufficient and physically plausible condition is that $\boldsymbol{\nabla} \mathbf{v} \geq C>0$ everywhere in the area enclosed by the homoclinic trajectory in Fig. 1 with $C$ independent of $\varepsilon, \eta$. We note that $\boldsymbol{\nabla} \mathbf{v}$ must be positive at the point $\left(q_{a}, p_{a}\right)$ in order for the state $\left(q_{a}, p_{a}\right)$ to be stable.

Close to a nonlocal Hamiltonian bifurcation point $|p| \sim \eta^{1 / 2} \ll 1$, and therefore, generically, we can expand $\mathbf{v}(p, q)$ in $p$. The term $\propto p$ in $v^{(q)}$ can be disregarded, and it suffices to keep only a linear in $p$ term in $v^{(p)}$; the $p$-independent term in $v^{(p)}$ can be incorporated into the Hamiltonian leading to a trivial renormalization $U(q) \rightarrow U(q)+(\varepsilon / \eta) v^{(p)}(p=0, q)$. Then the stationary states in the presence of friction are given by the equations

$$
\begin{aligned}
& p=\varepsilon v^{(q)} \\
& \eta \partial_{q} U(q)+\varepsilon^{2}\left[v^{(q)} \partial_{p} v^{(p)}\right]_{p=0}=0 .
\end{aligned}
$$

The positions of the states remain practically unchanged with the varying parameter of the Hamiltonian $\eta$ as long as $\eta \gg \varepsilon^{2}$. When this condition holds, the frequency of vibrations near the attractor $\omega_{a} \sim \eta^{1 / 2}$ largely exceeds their decay rate $\propto \varepsilon$ and the motion is underdamped, as expected.

If, as we assume, the system displays a saddle-node bifurcation for a small $\eta$, then with decreasing $\eta$ the stationary states come closer to each other and ultimately merge together for $\eta=\eta_{B}^{\mathrm{nl}} \sim \varepsilon^{2}$. Operationally, the bifurcation value of the control parameter $\eta_{B}^{\mathrm{nl}}$ is determined by the condition that two solutions of Eq. (17) for $q, p$ coincide. An illustration for a specific model is provided below.

We note that Eq. (17) may have two solutions for $\eta_{B}^{\mathrm{nl}}$. This means that there are two bifurcation points in the region of small $|\eta|$. The possibility of such behavior is clear from the Hamiltonian (12). For example, for $U(q)$ of the type of a cubic parabola, the patterns of trajectories for positive and negative $\eta$ are similar, with the center and the hyperbolic point interchanged. The two values of $\eta_{B}^{\mathrm{nl}}$ correspond to the saddle-node bifurcations of these two types of motion with decreasing $|\eta|$ in the presence of friction.

Eq. (16) describes energy drift in the case of a local bifurcation as well. In this case, from Eq. (14), $\boldsymbol{\nabla} \mathbf{v}=a_{p}+a_{q}$ is constant. The integral (16) can be found explicitly, in terms of elliptic functions.

\section{ENERGY DIFFUSION}

A major effect of noise on the dynamics of underdamped systems is drift and diffusion of energy. Their rates are determined by the noise intensity parameters $D_{i j}$ (2). In the weak-noise limit, where $D_{i j}$ are the smallest parameters of the theory, noise-induced energy drift can be disregarded compared to the drift induced by fric- 
tion. Therefore we will be interested only in energy diffusion.

The period-average value $D_{E}$ of the energy diffusion coefficient can be obtained from the equation of motion (11) in a standard way: we write $d H / d t$ as $\partial_{q} H \dot{q}+\partial_{p} H \dot{p}$, substitute the noise terms $f^{(q)}$ and $f^{(p)}$ for $\dot{q}$ and $\dot{p}$, find the correlator of $d H / d t$ using the noise correlators (21), and average the corresponding diffusion coefficient over the period $2 \pi / \omega(E)$. Changing at the last step from time integration to integration along the trajectory $H(p, q ; \eta)=E$, we obtain

$$
\begin{aligned}
D_{E}= & \frac{\omega(E)}{2 \pi} \oint\left[d q\left(D_{p p} \partial_{p} H+D_{p q} \partial_{q} H\right)-d p\left(D_{q q} \partial_{q} H+D_{p q} \partial_{p} H\right)\right] \\
= & \frac{\omega(E)}{2 \pi} \iint_{A(E)} d q d p\left[\partial_{p}\left(D_{p p} \partial_{p} H+D_{p q} \partial_{q} H\right)+\partial_{q}\left(D_{q q} \partial_{q} H+D_{p q} \partial_{p} H\right)\right]
\end{aligned}
$$

Near the bifurcation point the momentum $p$ is small, $p \propto \eta^{3 / 4}$ and $p \propto \eta^{1 / 2}$ for the local and nonlocal bifurcation, respectively. The derivatives $\partial_{q} H, \partial_{q}^{2} H$ are also small, with $\partial_{q}^{2} H \propto \eta^{1 / 2}$ and $\partial_{q}^{2} H \propto \eta$ for the local and nonlocal bifurcation. Then taking into account that $\partial_{p} H=p$, we can simplify Eq. [18],

$$
D_{E} \approx \frac{\omega(E)}{2 \pi} \iint_{A(E)} d q d p D_{p p} \partial_{p}^{2} H=\frac{\omega(E)}{2 \pi} \iint_{A(E)} d q d p D_{p p} .
$$

Here, we have assumed that the coefficient $D_{p p}$ is not proportional to a power of a small parameter $\eta$ inside the areas enclosed by the homoclinic orbits in Fig. 1

\section{ESCAPE RATE}

Diffusion over energy leads to escape from a metastable state. The escape rate can be calculated from the onedimensional Fokker-Planck equation for the energy distribution $\rho(E)$,

$$
\partial_{t} \rho=\partial_{E}\left(-V_{E} \rho+D_{E} \partial_{E} \rho\right)
$$

(here, again, we have disregarded the corrections $\propto D$ to the energy drift velocity).

The escape rate $W$ is determined by the probability density to have the energy of the saddle point (the top of the potential barrier) $E=E_{s}$, given that, for small noise intensity, the system is mostly fluctuating about the center, $E=E_{c}$. From Eq. (20) we have

$$
\begin{aligned}
& W=\text { const } \times \exp (-R / D), \\
& R=-\int_{E_{c}}^{E_{s}} d E V_{E} \frac{D}{D_{E}}
\end{aligned}
$$

(here, $D$ is the characteristic noise intensity, see below). Eq. (21) is central for the following calculation. It immediately allows us to find the scaling of the activation energy near a bifurcation point for the local and nonlocal bifurcation.

In the case of the local bifurcation the ratio $V_{E} / D_{E}$ can be obtained in the explicit form. From Eq. (14) we have $\boldsymbol{\nabla} \mathbf{v}=a_{p}+a_{q}$. Because the area of integration $A(E)$ in Eq. (19) is limited to a small vicinity of the point $q=$ $p=0$, we can set in Eq. (19) $D_{p p}(p, q) \approx D_{p p}(0,0) \equiv D$. Then from Eqs. (16), (19) we obtain $V_{E} / D_{E}=-\varepsilon\left(a_{p}+\right.$ $\left.a_{q}\right) / D$. The activation energy of escape $R_{\text {loc }}$ is simply proportional to the energy difference $\Delta E=E_{s}-E_{c}$ (11),

$$
R_{\mathrm{loc}}=\varepsilon\left(a_{p}+a_{q}\right) \Delta E=\frac{4}{3} \varepsilon\left(a_{p}+a_{q}\right) \eta^{3 / 2} .
$$

Eq. (22) shows that, for the local bifurcation, in the general case of a nonequilibrium underdamped system the activation energy of escape scales with the distance to the bifurcation point as $\eta^{\xi}$ with $\xi=3 / 2$. This is the same exponent as in the overdamped regime.

In the case of the nonlocal Hamiltonian bifurcation the range of integration over $p$ in the expressions (16), (19) for $V_{E}$ and $D_{E}$ is narrow, $\propto \eta^{1 / 2}$, whereas the range of integration over $q$ is independent of $\eta$. Therefore the integrands $\boldsymbol{\nabla} \mathbf{v}$ and $D_{p p}$ in these expressions can be calculated for $p=0$. Then integration over $p$ can be done directly, and the integrals in Eqs. (16) and (19) take a form

$$
\begin{aligned}
& \left(E_{s}-E_{c}\right)^{1 / 2} \int_{q_{\min }(x)}^{q_{\max }(x)} d q f(q) \\
& \times\left\{x-\frac{\eta}{E_{s}-E_{c}}\left[U(q)-U\left(q_{c}\right)\right]\right\}^{1 / 2},
\end{aligned}
$$

where $x \equiv x(E)=\left(E-E_{c}\right) /\left(E_{s}-E_{c}\right)$ is the reduced energy, and $f(q)$ is proportional to $\boldsymbol{\nabla} \mathbf{v}(p=0, q)$ and $D_{p p}(p=0, q)$, respectively. The functions $q_{\min }$ and $q_{\max }$ determine the limits of integration and are given by the equation $E=\eta U(q)$.

Since $\Delta E=E_{s}-E_{c}=$ const $\times \eta[$ cf. Eq. [13)], the expression (23) has the form $\left(E_{s}-E_{c}\right)^{1 / 2} \tilde{f}(x)$, i.e., it 
depends on energy only in terms of the parameter $x(E)$ and is proportional to $\eta^{1 / 2}$. Therefore the ratio $V_{E} / D_{E}$ is a function of $\left(E-E_{c}\right) /\left(E_{s}-E_{c}\right)$, which is proportional to $\varepsilon / D$, with $D$ being the characteristic noise intensity. Then, from Eq. (21), to leading order in $\eta$ the activation energy near the nonlocal bifurcation $R_{\mathrm{n}}$ is proportional to the energy difference $\Delta E \propto \eta$, as in the local case,

$$
R_{\mathrm{nl}}=\text { const } \times \varepsilon \eta .
$$

From Eq. (24) the activation energy scales with the distance to the bifurcation point as $\eta^{\xi}$ with $\xi=1$. We are not aware of a prediction of such dependence in the general case. It is much slower than for the local Hamiltonian bifurcation.

\section{EXAMPLE: A RESONANTLY DRIVEN NONLINEAR OSCILLATOR}

A system where the both types of bifurcations may occur and which has recently attracted significant interest [3, 7, 8, 9] is an underdamped nonlinear oscillator driven by a resonant field. The motion of such an oscillator in the rotating frame is described by Eqs. (1) with

$$
\begin{aligned}
H(p, q) & =\frac{1}{4}\left(q^{2}+p^{2}-1\right)^{2}-\beta^{1 / 2} q, \\
v^{(q)} & =q, \quad v^{(p)}=p .
\end{aligned}
$$

For several microscopic and phenomenological models of noise, which include those of interest for the experiment [3, 7, 8, 9], the components $f^{(q)}, f^{(p)}$ are independent and $\delta$-correlated, with coordinate-independent $D_{p p}=D_{q q}=$ $D, D_{p q}=0$.

The parameter $\beta$ characterizes the intensity of the driving resonant field. For a small friction coefficient $\varepsilon$, the oscillator is bistable in the region $\beta_{B}^{(1)}<\beta<\beta_{B}^{(2)}$, where $\beta_{B}^{(1)} \approx \varepsilon^{2}$ and $\beta_{B}^{(2)} \approx 4 / 27$.

The Hamiltonian $H$ is shown in Fig. [2 It has the shape of a tilted Mexican hat. The local maximum (the top of the internal dome) $A_{1}$ and the absolute minimum $A_{2}$ are centers; they become attractors due to friction; $S$ is the saddle point. A general expression for the escape rate of the oscillator was obtained in Ref. 19; in particular, the activation energy was calculated explicitly as a function of $\beta$ in the underdamped case $\varepsilon \ll 1$.

For the bifurcation value of the control parameter $\beta=\beta_{B}^{(2)}=4 / 27$ in the limit of small damping, the saddle point $S$ in Fig. 2 merges with the dome top $A_{1}$ at $q_{B}^{(2)}=-1 / \sqrt{3}, p=0$. For $\eta=(4 / 27)^{1 / 2}-\beta^{1 / 2} \ll 1$ and small $\delta q=q-q_{B}^{(2)}, p$, to the leading order in $\delta q, p, \eta$ the Hamiltonian (25) has the form

$$
H \approx-\frac{1}{3} p^{2}-\frac{1}{\sqrt{3}}(\delta q)^{3}+\eta(\delta q)+\text { const. }
$$

This expression has essentially the same form as the Hamiltonian (9) near the local bifurcation point. Note,

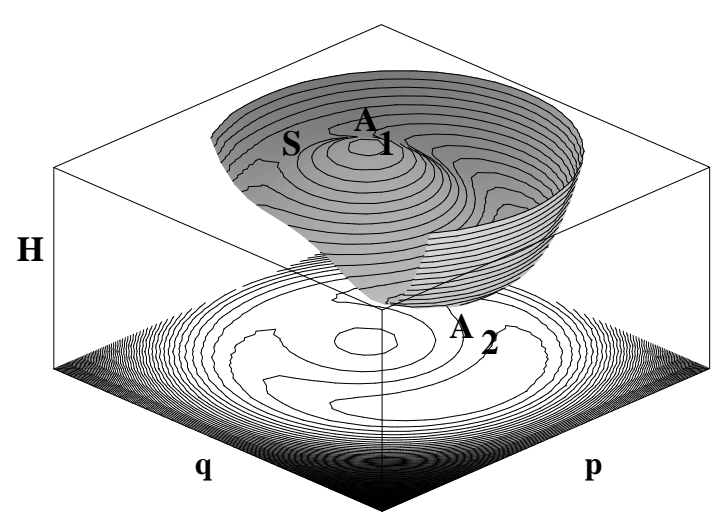

FIG. 2: The effective Hamiltonian $H(p, q)$ of a resonantly driven nonlinear oscillator in the rotating frame 25]. The plot refers to the reduced field $\beta=2 / 27$. The local maximum $A_{1}$ and the minimum $A_{2}$ are the centers, and $S$ is the saddle point. The trajectories for constant energy $H(p, q)=E$ are shown in the lower panel.

however, that the center is the local maximum rather than the minimum of the potential.

It follows from Eq. 26] that near the local bifurcation point $|\Delta E|=\left|E_{s}-E_{c}\right|=\left(4 / 3^{5 / 4}\right) \eta^{3 / 2}$. Then from Eq. 22]

$$
R_{\mathrm{loc}} \equiv R_{B}^{(2)}=\left(4 / 3^{1 / 4}\right) \varepsilon \eta^{3 / 2}=\frac{9 \varepsilon}{2}\left(\beta_{B}^{(2)}-\beta\right)^{3 / 2}
$$

(we have taken into account that, for the Hamiltonian (26), the last term in Eq. (19) has an extra factor 3/2).

Eq. (27) coincides with the expression obtained for the present system by Dmitriev and Dyakonov [17]. It coincides also with the expression obtained from the analysis of the oscillator dynamics very close to the bifurcation point $\beta_{B}^{(2)}$, where the oscillator motion is overdamped [10]. This is similar to the situation in equilibrium systems, where the escape rate as a function of the distance to the bifurcation point is given by the same expression in the opposite limits of underdamped and overdamped motion.

The dynamics of the oscillator near the bifurcation point $\beta_{B}^{(1)}$ is described by the nonlocal bifurcation theory. The structure of the phase portrait in the oscillator variables $(q, p)$ for the appropriate energy range is clear from Fig. [3] The homoclinic trajectories form a double loop with narrow space between the trajectories. The trajectories that surround the center $A_{2}$ have the shape of horseshoes "squeezed" into the interloop space, as seen also from Fig. 2

The slowing down of motion is a result of the fact that the system moves in the opposite directions along the homoclinic trajectories. When they approach each other (and the circle $p^{2}+q^{2}=1$ that lies between them) with $\beta$ approaching $\beta_{B}^{(1)}$, the motion is slowed down everywhere between them, including the center. 


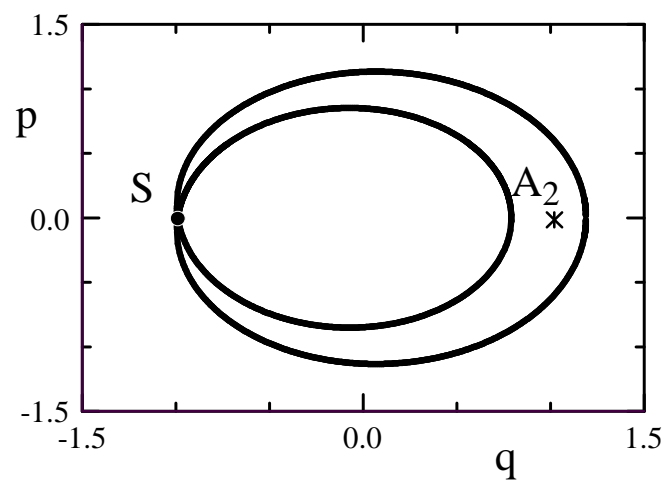

FIG. 3: Homoclinic trajectories of a resonantly driven oscillator near a nonlocal Hamiltonian bifurcation. The center $A_{2}$ is marked by the star, $S$ is the saddle point. As the control parameter $\beta$ approaches the bifurcation value $\beta_{B}^{(1)}$ the homoclinic trajectories approach each other and ultimately merge at the bifurcation point. At this time the energies of the saddle and the center become equal to each other. The data refer to $\beta=3.9 \times 10^{-4}$.

Even though the phase portrait in Fig. 3 looks somewhat different from the one in the lower panel of Fig. 1 they can be mapped onto each other. Therefore the results for escape near a nonlocal bifurcation should apply to the oscillator.

For $\beta \ll 1$ and $\left|p^{2}+q^{2}-1\right| \ll 1$ it is convenient to change in Eq. 25I to new canonical variables, $P=$ $-\left(p^{2}+q^{2}-1\right) / 2$ and $Q=\arctan (p / q)$. In these variables, to leading order in $P, \beta$ the Hamiltonian takes the form (12),

$$
H=P^{2}-\eta \cos Q, \quad \eta=\beta^{1 / 2} .
$$

The center and the saddle point are $Q_{c}=0, P_{c}=0$ and $Q_{s}=\pi, P_{s}=0$, respectively. The energy difference $\Delta E=E_{s}-E_{c}=2 \eta$.

From Eq. 25) and the expressions for the noise diffusion coefficients, the corresponding functions that describe friction force and noise in variables $Q, P$ are

$$
\begin{array}{cl}
v^{(P)}=1-2 P, & v^{(Q)}=D_{P Q}=0, \\
D_{P P}=D(1-2 P), & D_{Q Q}=D(1-2 P)^{-1} .
\end{array}
$$

We note that the oscillator has only one saddle-node bifurcation point for $\beta=\eta^{2} \ll 1$, because by construction $\eta>0$.

From Eqs. (16), (19), (29) we obtain $V_{E} / D_{E}=\varepsilon / D$. Therefore the activation energy of escape near the nonlocal bifurcation is

$$
R_{\mathrm{nl}} \equiv R_{B}^{(1)}=2 \varepsilon \eta=2 \varepsilon \beta^{1 / 2} .
$$

Eq. (30) coincides with the expression in Ref. 19 obtained by a completely different method.

\section{DISCUSSION OF RESULTS}

We have considered activated escape in systems close to a saddle-node bifurcation point, the problem of interest not only for recent experiments in different fields of physics [3, 7, 8, 9, 21], but also for such areas of recent attention as epidemic control [22]. Of special interest to us were underdamped systems. We studied the range of the control parameter where a system is close, but not too close to the bifurcation point. In this range the motion is already slowed down and is described by one degree of freedom, but is still underdamped. The slowness of motion justifies describing it by a Langevin equation in which delay of the friction force and finite correlation time of noise are disregarded.

We have identified two scenarios which an underdamped system can follow as it approaches a saddle-node bifurcation. One is local, where in the neglect of damping the system has a saddle point and a center close to each other in phase space. As the control parameter $\eta$ approaches the bifurcation value $\eta_{B}=0$ these states approach each other. It is the closeness of the states that leads to motion slowing down in this case. The second scenario is nonlocal, where the saddle point and the center remain far from each other until damping becomes strong. However, the phase trajectories have a shape of narrow cigars, and since on the opposite sides of the cigar the system moves in opposite directions, the overall motion becomes slow. As $\eta \rightarrow \eta_{B}$, the cigars are further squeezed, leading to further slowing down.

The major effect of friction is energy drift towards a stable state. In contrast, noise leads to energy diffusion away from the metastable state and ultimately to escape. The activation energy of escape $R$ is determined by the ratio of the drift and diffusion coefficients. We found that, for both bifurcation scenarios, this ratio has a simple form. It can be found explicitly for the local scenario, whereas for the nonlocal scenario it has a form of a function of reduced energy. As a result, the activation energy is proportional to the energy difference in the saddle point and the center.

We have shown that, for both types of underdamped systems, the activation energy displays scaling $R \propto \eta^{\xi}$. The scaling exponent $\xi=3 / 2$ for the local scenario and $\xi=1$ for the nonlocal one. The scaling $\xi=3 / 2$ has been known for a saddle-node bifurcation in overdamped systems and also for escape of a Brownian particle due to thermal fluctuations. As we show, this behavior is independent of the nature of friction and fluctuations and occurs even in the absence of detailed balance. The scaling $\xi=1$ is a new result. We give an example of a system of recent interest where both types of scaling can be observed.

MID acknowledges support by the NSF DMR-0305746, IBS acknowledges support from the Office of Naval Research, MS acknowledges support by the NSF DMS0401178 and BSF 2002375. The research of MID and MS was also supported in part by the Institute for Quantum 
Sciences at Michigan State University.

[1] T.A. Fulton and L.N. Dunkelberger, Phys. Rev. B 9, 4760-4768 (1974).

[2] M.H. Devoret, D. Esteve, J.M. Martinis, A. Cleland, and J. Clarke, Phys. Rev. B 36, 58-73 (1987).

[3] I. Siddiqi, R. Vijay, F. Pierre, C. M. Wilson, M. Metcalfe, C. Rigetti, L. Frunzio, and M. H. Devoret, Phys. Rev. Lett. 93, 207002 (2004).

[4] W. Wernsdorfer, E.B. Orozco, K. Hasselbach, A. Benoit, B. Barbara, N. Demoncy, A. Loiseau, H. Pascard, and D. Mailly, Phys. Rev. Lett. 78, 1791 (1997); W.T. Coffey, D.S.F. Crothers, J.L. Dormann, Yu.P. Kalmykov, E.C. Kennedy, and W. Wernsdorfer, Phys. Rev. Lett. 80, 5655 (1998).

[5] R.H. Koch, G. Grinstein, G.A. Keefe, Yu Lu, P.L. Trouilloud, and W.J. Gallagher, Phys. Rev. Lett. 84, 5419 (2000).

[6] E. B. Myers, F. J. Albert, J. C. Sankey, E. Bonet, R. A. Buhrman, and D. C. Ralph, Phys. Rev. Lett. 89, 196801 (2002).

[7] J. S. Aldridge and A. N. Cleland, Phys. Rev. Lett. 94 (2005); cond-mat/0406528 (2004).

[8] R. L. Badzey, G. Zolfagharkhani, A. Gaidarzhy, and P. Mohanty, Appl. Phys. Lett. 86, 023106 (2005).

[9] C. Stambaugh and H. B. Chan, cond-mat/0504791 (2005).

[10] M.I. Dykman and M.A. Krivoglaz, Physica 104A, 480
(1980).

[11] R. Graham and T. Tél, Phys. Rev. A 35, 1328 (1987).

[12] [M.I. Dykman, E. Mori, J. Ross, and P. Hunt, J. Chem. Phys. 100, pp. 5735 - 50 (1994).

[13] O.A. Tretiakov, T. Gramerspacher, and K.A. Matveev, Phys. Rev. B 67, 073303 (2003); O.A. Tretiakov and K.A. Matveev, Phys. Rev. B 71, 165326 (2005).

[14] L.D. Landau and E.M. Lifshitz, Statistical Physics, pt. 1, 3rd edition revised by E.M. Lifshitz and L.P. Pitaevskii (Pergamon, Oxford 1980).

[15] H. Kramers, Physica (Utrecht) 7, 240 (1940).

[16] J. Kurkijärvi, Phys. Rev. B 6, 832-835 (1972).

[17] A.P. Dmitriev and M.I. D'yakonov, Zh. Eks. Teor. Fiz. 90, 1430 (1986) [Sov. Phys. JETP 63, 838 (1986)].

[18] R.H. Victora, Phys. Rev. Lett. 63, 457 (1989).

[19] M.I. Dykman and M.A. Krivoglaz, Zh. Eksp. Teor. Fiz. 77, 60 (1979) [Sov. Phys. JETP 50, 30 (1979).

[20] J. Guckenheimer and P. Holmes, Nonlinear Oscillators, Dynamical Systems and Bifurcations of Vector Fields (Springer-Verlag, NY 1987); V. Arnold, Ordinary Differential Equations, 3rd ed. (Springer, New York 1992).

[21] L. Billings, . B. Shwartz, D. S. Morgan, et al, Phys. Rev. E. 70, 026220 (2004).

[22] I. B. Schwartz, Phys. Rev. E 70, 046220 (2004). 